\title{
A NOTE ON POINT-COUNTABILITY IN LINEARLY ORDERED SPACES
}

\author{
HAROLD R. BENNETT
}

\begin{abstract}
In this note linearly ordered topological spaces (abbreviated LOTS) with a point-countable base are examined. It is shown that a LOTS is quasi-developable if and only if it has a $\sigma$-point-finite base and a LOTS with a point-countable base is paracompact. An example of a LOTS with a point-countable base that does not have a $\sigma$-point-finite base is given. Conditions are given for the metrizability of a LOTS with a point-countable base and it is shown that a connected LOTS with a point-countable base is homeomorphic to a connected subset of the real line.
\end{abstract}

1. Introduction. Recent years have seen a growth of interest in topological spaces which have a point-countable base [1]. For example, Miščenko proved that a compact Hausdorff space with a point-countable base is metrizable [20] and Heath generalized this result by showing that a metacompact $p$-space with a point-countable base is developable [15]. In a different direction, Heath has also shown that a semimetric space with a point-countable base is developable [14].

A particularly interesting subclass of the class of spaces with a point-countable base is the class of spaces with a $\sigma$-point-finite base. These spaces have been studied by S. Hanai [12], C. E. Aull [4] and Heath [13]. In [3], Arhangel'skil showed that a perfectly normal, collectionwise normal space with a $\sigma$-point-finite base is metrizable.

In this note linearly ordered topological spaces which have a pointcountable base are examined. Linearly ordered spaces with a $\sigma$-pointfinite base are characterized in terms of quasi-developability and an example is given of a linearly ordered space with a point-countable base that does not have a $\sigma$-point-finite base.

2. Point-countability in linearly ordered spaces. Recall that a linearly ordered topological space (abbreviated LOTS) is a topological space whose topology agrees with the topology induced by some linear ordering. (Throughout this note let $<$ denote the linear ordering.) Also recall that if $B$ is a collection of subsets of a set $X$, then $Q$ is said to be a point-countable (point-finite) collection if each

Received by the editors March 23, 1970 and, in revised form, April 24, 1970.

AMS 1969 subject classifications. Primary 5440; Secondary 5435, 5438, 5450, 5455.

Key words and phrases. Linearly ordered spaces, point-countable base, $\sigma$-pointfinite base, quasi-developable space. 
element of $X$ is in at most countably (finitely) many elements of $B$ and $B$ is said to be a $\sigma$-point-finite collection if $B=\cup\left\{B_{i}: i \in Z^{+}\right\}$ where each $B_{i}$ is a point-finite collection. A base $B$ for a space $X$ is said to be a point-countable base ( $\sigma$-point-finite base) if $B$ is a pointcountable ( $\sigma$-point-finite) collection.

Let all undefined terms and notation be as in [7]. In particular let $E^{1}$ denote the set of real numbers and $Z^{+}$denote the set of natural numbers.

One of the most useful tools in studying collections of intervals in a LOTS is the notion of a coherent collection of convex sets.

(2.1) Definition. A collection of sets $a$ is said to be a coherent collection if, for any subcollection $B$ of $Q$, there is an element of $B$ that intersects some element of $a-B$. The collection $B$ is said to be a maximal coherent subcollection of $Q$ if there does not exist a coherent subcollection $\mathcal{C}$ of $\mathfrak{a}$ such that $\mathbb{B}$ is a proper subcollection of $\mathfrak{C}$.

If $\mathcal{G}$ is a collection of sets, then let $\mathcal{G}^{*}=\bigcup\{G \in \mathcal{G}\}$.

The following lemma is obvious.

(2.2) LeMмA. Let $\mathcal{G}$ be a collection of nonempty subsets of a set $X$. Let $\mathfrak{C}$ be the family of all maximal (with respect to $C$ ) coherent subcollections of $\mathcal{S}$. Then $\mathcal{G}=\mathrm{U} \mathcal{C}$ and if $\mathcal{H C}$ and $\mathfrak{K}$ are distinct elements of $\mathcal{C}$, then $\mathfrak{H}^{*} \cap \mathfrak{K}^{*}=\varnothing$.

(2.3) Definition. A subset $A$ of a linearly ordered set $X$ is said to be convex if whenever $a, b \in A$, then $\{x \in X: a<x<b\} \subseteq A$.

The next lemma is fundamental in proving the results of this section.

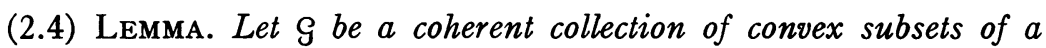
linearly ordered set $X$. Then there are simple sequences $\{x(i)\}$ and $\{y(i)\}$, possibly finite, of points of $\mathcal{G}^{*}$ such that

(a) $x(1)=y(1)$,

(b) if $x(k+1)$ (respectively $y(k+1)$ ) is defined, then $x(k+1)<x(k)$ (respectively $y(k)<y(k+1)$ ) and $x(k+1) \notin \operatorname{St}(x(k), \mathcal{G})$ (respectively $y(k+1) \notin \operatorname{St}(y(k), \mathcal{G}))$ and $\operatorname{St}(x(k), \mathcal{G}) \cap \operatorname{St}(x(k+1), \mathcal{G}) \notin \varnothing$, (respectively $\operatorname{St}(y(k), \xi) \cap \operatorname{St}(y(k+1), \mathcal{S}) \notin \varnothing)$, and

(c) each element of $\mathcal{G}^{*}$ is contained in some set $\operatorname{St}(x(k), \mathcal{G})$ or some set $\operatorname{St}(y(k), \mathcal{S})$.

Proof. Let $\alpha$ be a well ordering of $\mathcal{G}^{*}$ and let $x(1)$ be the first element of $\alpha$. If $x(1), \cdots, x(k)$ have been defined, let $x(k+1)$, if it exists, be the first element of $\alpha$ such that

(i) $x(k+1)<x(k)$,

(ii) $x(k+1) \notin \operatorname{St}(x(k), \xi)$, and 
(iii) $\operatorname{St}(x(k+1), \varsigma) \cap \operatorname{St}(x(k), \varsigma) \neq \varnothing$.

It follows that there can be at most a simple sequence of such points for suppose $\mathcal{G}^{*}$ contains an element $x(\infty)$ such that

(i) $x(\infty)<x(k)$ for each $k \in Z^{+}$,

(ii) $x(\infty) \notin \operatorname{St}(x(k), \mathcal{G})$ for each $k \in Z^{+}$, and

(iii) $\operatorname{St}(x(\infty), \mathcal{\varsigma}) \cap\left(U\left\{\operatorname{St}(x(k), \mathcal{\varsigma}): k \in Z^{+}\right\}\right) \neq \varnothing$.

Hence there exists $k \in Z^{+}$such that $\operatorname{St}(x(k), \S) \cap \operatorname{St}(x(\infty), \S) \neq \varnothing$. But then it follows that $x(\infty) \in \operatorname{St}(x(k+1), \S)$ which is a contradiction. Let $x(1)=y(1)$ and, in an analogous manner, choose a simple sequence $\{y(k)\}$ such that

(i) $y(k)<y(k+1)$,

(ii) $y(k+1) \notin \operatorname{St}(y(k)$, 乌), and

(iii) $\operatorname{St}(y(k), \mathcal{g}) \cap \operatorname{St}(y(k+1), \mathcal{g}) \neq \varnothing$.

It follows that $\{x(k)\}$ and $\{y(k)\}$ are the desired sequences.

The following corollary is an immediate consequence of parts (b) and (c) of the preceding lemma.

(2.5) Corollary. If $\mathcal{G}$ is a point-countable coherent collection of convex subsets of a linearly ordered set $X$, then there is a countable subcollection $\mathfrak{H C}$ of $\mathcal{G}$ such that $\mathfrak{H C}^{*}=\mathrm{G}^{*}$. [8]).

The next theorem was proved independently ${ }^{1}$ by Fedorčuk (see

(2.6) ThEOREM. A LOTS is paracompact if every open cover has an open point-countable refinement.

Proof. Let $X$ be a LOTS with the property that each open cover has an open point-countable refinement. Let $\mathcal{u}$ be any open covering of $X$ and let $\mathcal{G}$ be an open point-countable refinement of $u$. No gener-

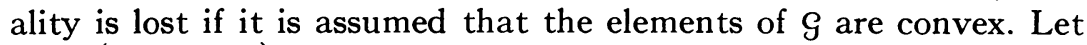
$\mathcal{G}=\cup\left\{\mathfrak{e}_{\alpha}: \alpha \in A\right\}$ where each $\mathfrak{e}_{\alpha}$ is a maximal coherent subcollection of $\mathrm{g}$. By (2.2) if $\alpha \neq \beta$, then $\mathfrak{e}_{\alpha}^{*} \cap \mathfrak{e}_{\beta}^{*}=\varnothing$ and, for each $\alpha \in A$, there is a countable subcollection $\mathfrak{H}_{\alpha}=\left\{H(\alpha, i): i \in Z^{+}\right\}$such that $\mathfrak{K}_{\alpha}^{*}=\mathfrak{C}_{\alpha}^{*}$. It follows that $\mathfrak{K}_{i}=\{H(\alpha, i): \alpha \in A\}$ is a locally finite collection and $\mathscr{K}=U\left\{\mathcal{K}_{i}: i \in Z^{+}\right\}$is a $\sigma$-locally finite open refinement of $\mathcal{u}$. Thus $X$ is paracompact.

(2.7) Corollary. A LOTS with a point-countable base is hereditarily paracompact.

Proof. Let $X$ be a LOTS with a point-countable base. Note that each open subspace of $X$ is the pairwise disjoint union of its convex

${ }^{1}$ This was pointed out by the referee to whom the author is very grateful for valuable suggestions in completing this note. 
components, each of which is open. It follows that each of the convex components is paracompact since each is a LOTS in its relative topology and has a point-countable base. Thus each open subspace of $X$ is paracompact. Theorem 2.5(b) of [7] shows that this is suffcient to insure that $X$ is paracompact.

The concept of a quasi-developable space is a natural generalization of a developable space (see [21]). A LOTS with a $\sigma$-pointfinite base can be characterized in terms of the concept of quasidevelopability.

(2.8) Definition. A sequence $\left\{g_{1}, g_{2}, \ldots\right\}$ of collections of open subsets of a topological space $X$ is a quasi-development for $X$ provided for each $p \in X$ and each open set $R$ containing $p$ there exists $n(p, R) \in Z^{+}$such that $p \in \operatorname{St}\left(p, S_{n(p, R)}\right) \subset R$. A topological space with a quasi-development is called a quasi-developable space.

The next example shows a space, even a LOTS, may be quasidevelopable without being developable.

(2.9) ExAmple. Let $Y$ denote the unit square topologized by the lexicographic ordering (see [16]) and let $Y$ contain $X=\{(x, y)$ $\in Y$ :if $x \in Q$, then $y=0$ or $y=1$ or if $x \in E^{1}$, then $y=1 / n$ or $y=1-1 / n$, for $\left.n \in Z^{+}, n \geqq 2\right\}$. It follows that $X$ topologized by the induced lexicographic ordering is a nonmetrizable quasi-developable LOTS. Let $\mathcal{S}_{1}=\left\{\{(x, y)\}: x \in E^{1}\right.$ and $\left.y \notin\{0,1\}\right\}$ and if $\left\{R_{1}, R_{2}, \cdots\right\}$ is a countable base for the countable set $T=\{(x, y): x \in Q$ and $y \in\{0,1\}\}$, then let $g_{n+1}=\left\{R_{n}\right\}$ for each $n \in Z^{+}$. It follows that $\left\{g_{1}, \mathcal{G}_{2}, \cdots\right\}$ is a quasi-development for $X$. Since $T$ is a closed, non- $G_{\delta}$-subset of $X$, it is clear that $X$ is nonmetrizable.

In [4], C. E. Aull has shown that a space with a $\sigma$-point-finite base is quasi-developable. Example 3.3 of [19] shows that, in general, the converse of this result is not true, but for a LOTS the following holds.

(2.10) TheOREM. A LOTS is quasi-developable if and only if it has a $\sigma$-point-finite base.

Proof. Let $X$ be a LOTS with quasi-development $\left\{g_{1}, g_{2}, \cdots\right\}$. Without loss of generality let the elements of each $g_{n}$ be convex sets. If $n \in Z^{+}$, let $\left\{g(n, \alpha): \alpha \in A_{n}\right\}$ be the set of all maximal coherent subcollections of $\mathcal{G}_{n}$. Let $H(n, \alpha)=\{x(1), x(2), \cdots, y(1), y(2), \cdots\}$ be the countable collection of points of $\mathcal{G}(n, \alpha)^{*}$ described in (2.4). Let $H_{n}=\bigcup\left\{H(n, \alpha): \alpha \in A_{n}\right\}$. For each $x \in H_{n}$ construct the following sets

(i) $\operatorname{St}\left(x, \mathcal{G}_{n}\right)$,

(ii) $\operatorname{RSt}\left(x, \Theta_{n}\right)=\left\{y \in \operatorname{St}\left(x, \varrho_{n}\right): x<y\right\}$, and

(iii) $\operatorname{LSt}\left(x, \Theta_{n}\right)=\left\{y \in \operatorname{St}\left(x, S_{n}\right): y<x\right\}$.

Let 
$\mathrm{B}_{n}=\left\{\operatorname{St}\left(x, G_{n}\right): x \in H_{n}\right\} \cup\left\{\operatorname{RSt}\left(x, \varrho_{n}\right): x \in H_{n}\right\} \cup\left\{\operatorname{LSt}\left(x, G_{n}\right): x \in H_{n}\right\}$.

Notice that $\Theta_{n}$ is a point-finite collection for if $p \in \mathbb{B}_{n}^{*}$, then either $p \in H_{n}$ or $p \notin H_{n}$. If $p \in H_{n}$, then $p$ is only in $\operatorname{St}\left(p, \mathcal{G}_{n}\right)$. If $p \notin H_{n}$, then there exists $z \in H_{n}($ let $z<p)$ such that $p \in \operatorname{St}\left(z, S_{n}\right)$. If there is a $t \in H_{n}$ such that $z<t$ and $\operatorname{St}\left(z, \mathcal{G}_{n}\right) \cap \operatorname{St}\left(t, \mathcal{S}_{n}\right) \neq \varnothing$, then $p$ is in at most four elements of $\otimes_{n}$, namely $\operatorname{St}\left(z, G_{n}\right), \operatorname{RSt}\left(z, S_{n}\right), \operatorname{LSt}\left(t, G_{n}\right)$, and $\operatorname{St}\left(t, S_{n}\right)$. If no such $t$ exists, then $p$ is in at most $\operatorname{St}\left(z, S_{n}\right)$ and $\operatorname{RSt}\left(z, S_{n}\right)$.

Define $B_{n}$ for each $n \in Z^{+}$and let $B=\bigcup\left\{\wp_{n}: n \in Z^{+}\right\}$. It follows that $B$ is a base for $X$, for if $p \in X$ and $R$ is an open set containing $p$, then there exists $n(p, R)=n \in Z^{+}$such that $p \in \operatorname{St}\left(p, G_{n}\right) \subset R$. If $p \in H_{n}$, then $\operatorname{St}\left(p, S_{n}\right) \in B_{n}$ and $B$ is a base. If $p \notin H_{n}$, then there is a $z \in H_{n}$ (let $z<p)$ such that $p \in \operatorname{St}\left(z, g_{n}\right)$. It follows that $\operatorname{RSt}\left(z, g_{n}\right) \subset \operatorname{St}\left(p, g_{n}\right)$ $\subset R$. Since $\operatorname{RSt}\left(z, g_{n}\right) \in \Theta_{n}$, it is clear that $B$ is the desired $\sigma$-pointfinite base for $X$.

It is clear that a space with a $\sigma$-point-finite base has a pointcountable base. The next example, an alteration of an example which M. E. Rudin was kind enough to communicate to the author, shows that the converse of this statement is not true even for LOTS.

(2.11) EXAMPLE. There is a hereditarily paracompact LOTS $X$ that has a point-countable base and does not have a $\sigma$-point-finite base. If $\omega_{1}$ denotes the first uncountable ordinal, then

$$
X=\left\{\left\{x_{\alpha}: \alpha \leqq \lambda\right\}: \lambda<\omega_{1}, x_{\alpha} \in E^{1}-Q \text { if } \alpha<\lambda, x_{\lambda} \in Q\right\} .
$$

If $x=\left\{x_{\alpha}: \alpha \leqq \lambda\right\}$ and $y=\left\{y_{\alpha}: \alpha \leqq \delta\right\}$ let $L(x)=\lambda$ and agree that $y$ extends $x$ if $x_{\alpha}=y_{\alpha}$ for each $\alpha<\lambda$. If $x \neq y$, then there is a first ordinal $\gamma$ such that $x_{\gamma} \neq y_{\gamma}$; define $x<y$ if $x_{\gamma}<y_{\gamma}$. The linear topology induced on $X$ by the linear ordering $<$ has the desired properties.

Let $U(x, n)=\left\{y \in X: y\right.$ extends $x$ and $x_{\lambda}-1 / n<y_{\lambda}<x_{\lambda}+1 / n$ where $\lambda=L(x)\}$ and let $\mathcal{U}=\left\{U(x, n): x \in X, n \in Z^{+}\right\}$. It is easy to check that $\mathcal{u}$ is a base for $X$. Since each $x \in X$ can extend at most countably many elements of $X$, it follows that $\mathcal{U}$ is a point-countable base for $X$.

To show that $X$ does not have a $\sigma$-point-finite base it is sufficient to show that if $B$ is a base for $X$, then $B$ has an uncountable, monotone decreasing (with respect to set inclusion) subfamily. If $B$ is any base for $X$, let $B_{1} \in B$ and $x_{1} \in B_{1}$. There exists $n_{1} \in Z^{+}$such that $U\left(x_{1}, n_{1}\right) \subset B_{1}$. Let $x_{2}$ be an extension of $x_{1}$ such that $x_{2} \in U\left(x_{1}, n_{1}\right)$ and $L\left(x_{2}\right)=L\left(x_{1}\right)+1$. Then there exists $B_{2} \in \Theta$ and $n_{2} \in Z^{+}$such that $U\left(x_{2}, n_{2}\right) \subset B_{2} \subset U\left(x_{1}, n_{1}\right)$. To apply transfinite induction suppose $x_{1}, \cdots, x_{\alpha}, \cdots$ and $B_{1}, \cdots, B_{\alpha}, \cdots$ have been chosen for each $\alpha<\beta<\omega_{1}$ such that for each $\alpha<\beta$

(i) $x_{\alpha}$ extends $x_{\gamma}$ if $\gamma<\alpha$, 
(ii) $U\left(x_{\alpha}, n_{\alpha}\right) \subset B_{\alpha}$, and

(iii) $B_{1} \supset U\left(x_{1}, n_{1}\right) \supset \cdots \supset B_{\alpha} \supset U\left(x_{\alpha}, n_{\alpha}\right) \supset \cdots$

Choose $x_{\beta} \in X$ such that $x_{\beta}$ extends $x_{\alpha}$ if $\alpha<\beta$ and

$$
L\left(x_{\beta}\right)=\sup \left\{L\left(x_{\alpha}\right): \alpha<\beta\right\}+1 .
$$

It follows $U\left(x_{\beta}, 1\right) \subset U\left(x_{\alpha}, n_{\alpha}\right)$ for each $\alpha<\beta$. Thus $U\left(x_{\beta}, 1\right) \subset B_{\alpha}$ for each $\alpha<\beta$. Let $B_{\beta} \in B$ such that $x_{\beta} \in B_{\beta} \subset U\left(x_{\beta}, 1\right)$. Then there exists $n_{\beta} \in Z^{+}$such that $U\left(x_{\beta}, n_{\beta}\right) \subset B_{\beta}$. Thus the induction step is complete and $B$ contains an uncountable, monotone decreasing subfamily $\left\{B_{\alpha}: \alpha<\omega_{1}\right\}$. Consequently $B$ is not a $\sigma$-point-finite base.

It follows from (2.7) that $X$ is hereditarily paracompact.

3. Metrization of LOTS with a point-countable base. A space $X$ satisfies the countable chain condition (abbreviated CCC) if any disjoint collection of open sets is countable. Since a LOTS which satisfies CCC is hereditarily Lindelöf [18], it follows from (2.10) that a LOTS with a $\sigma$-point-finite base which has $\mathrm{CCC}$ satisfies the Second Axiom of Countability. In [24], it is shown that a LOTS is collectionwise normal, so the theorem of Arhangel'skir [3] which was quoted in the introduction establishes the second part of the following theorem.

(3.1) TheOREM. Let $X$ be a LOTS with a $\sigma$-point-finite base. Then $X$ is metrizable if $X$ satisfies either of the following:

(i) $X$ satisfies $C C C$,

(ii) $X$ is perfectly normal.

If the above hypothesis is weakened to require that $X$ only has a point-countable base, then (3.1) can no longer be proved. This is shown in the next theorem which was proved independently ${ }^{1}$ by Ponomarev [22]. Recall that a Souslin space ${ }^{2}$ is a nonseparable LOTS which satisfies CCC. It is easily seen that a Souslin space has closed sets $G_{\delta}$.

(3.2) TheоRем. If there exists a Souslin space, then there is a Souslin space with a point countable base.

Proof. The proof (see [5]) is quite lengthy and will be omitted.

It would be interesting to know whether a perfectly normal LOTS with a point-countable base must be metrizable, independent of the Souslin hypothesis. Notice that the space in (2.10) does not have closed sets $G_{\delta}$.

If a completeness condition is imposed on the spaces in question, then metrization theorems can be obtained for LOTS with a point-

2 It is known to be consistent with certain axioms of set theory that such a space exists. See [23]. 
countable base. One such condition is that of a $p$-space in the sense of Arhangel'skiǐ [2].

(3.3) Definition. A completely regular space $X$ is a $p$-space provided that for some Hausdorff compactification $Y$ of $X$ there is a sequence $\left\{\rho_{1}, P_{2}, \cdots\right\}$ of collections of open subsets of $Y$, each of which covers $X$, such that if $x \in X$, then $\cap\left\{\operatorname{St}\left(x, \odot_{n}\right): n \in Z^{+}\right\} \subset X$.

It is known that any locally compact space and any absolute $G_{\delta}$ space (i.e. a space that is a $G_{\delta}$ subset of its Stone-Čech compactification) is a $p$-space.

(3.4) TheOrem. A LOTS is metrizable if and only if it is a p-space with a point-countable base.

Proof. Let $X$ be a LOTS with a point-countable base that is a $p$-space. By (2.6), $X$ is paracompact. In [9] it is shown that a paracompact $p$-space with a point-countable base is metrizable.

It is known [17] that any connected or locally connected LOTS is locally compact, so a (locally) connected LOTS with a point-countable base is metrizable. However more can be shown: a connected LOTS with a point-countable base is a subspace of the real line. The proof is based on a characterization of paracompactness in LOTS found in $[10]$ and it is convenient to repeat some definitions before proceeding.

(3.5) Definition. An interior gap of a LOTS $X$ is a Dedekind cut $(A \mid B)$ of $X$ such that $A$ has no last element and $B$ has no first element; such a gap is regarded as a virtual element of $X$ and it satisfies the expected ordering relationships. In case $X$ has no first element, a virtual element $u$, such that $u<x$ for all $x$ in $X$, is introduced and referred to as the left-end gap of $X$; if $X$ has no last element the rightend gap is defined analogously. The compact LOTS consisting of $X$ together with all its gaps is denoted by $X^{+}$.

(3.6) Definition. A gap $u$ of a LOTS $X$ is called a $Q$-gap from the left (right) provided there exists a regular initial ordinal $\omega_{\alpha}$ and an increasing (decreasing) sequence $\left\{x_{\gamma}: \gamma<\omega_{\alpha}\right\}$ of points of $X^{+}$having the limit $u$ such that for every limit ordinal $\lambda$ less than $\omega_{\alpha}$, the limit in $X^{+}$of $\{x: \gamma<\lambda\}$ is a gap of $X$; a gap $u$ of $X$ is called a $Q-g a p$ if it is a $Q$-gap from the left and from the right (or only the appropriate one in case $u$ is an end gap).

The characterization referred to is that a LOTS is paracompact if and only if every gap is a $Q$-gap.

(3.7) TheOREM. A connected LOTS with a point-countable base is homeomorphic to a connected subset of the real line. 
Proof. Let $X$ be a connected LOTS with a point-countable base. By (2.6), $X$ is paracompact and, thus, every gap of $X$ is a $Q$-gap. Observe then, since $X$ is connected, the regular initial ordinal associated with any gap must be $\omega_{0}$. The result follows by considering three cases.

If $X$ has both endpoints, then $X$ is a compact space with a pointcountable base. Thus $X$ is metrizable (see [19]) and, therefore, separable.

If $X$ has only one endpoint, say the right endpoint $b$, then the left endpoint $v$ is a virtual element of $X^{+}$and there is a point sequence $\left\{x_{1}, x_{2}, \cdots\right\}$ of elements of $X$ that converges to $v$. For each $n \in Z^{+}$, $\left[x_{n}, b\right]$ is a compact metric space and, thus, separable. Therefore $X=\bigcup\left\{\left[x_{n}, b\right]: n \in Z^{+}\right\}$is separable.

It is easy to generalize these arguments to the case where $X$ has no endpoints and see that, in any case, $X$ is separable. It follows, by a characterization in [11], that $X$ is homeomorphic to a connected subset of the real line.

Notice that the hypothesis of (3.7) cannot be weakened by replacing "connected" by "locally connected." To see this consider the LOTS $\{(x, y): 0 \leqq x \leqq 1,0<y<1\}$ ordered lexicographically.

\section{REFERENCES}

1. P. S. Alexandroff, Some results in the theory of topological spaces obtained within the last twenty-five years, Uspehi Mat. Nauk 15 (1960), no. 2 (92), 25-95=Russian Math. Surveys 15 (1960), no. 2, 23-83. MR 22 \#9947.

2. A. V. Arhangel'ski1, $A$ class of spaces containing all metric and all locally bicompact spaces, Dokl. Akad. Nauk SSSR 151 (1963), 751-754=Soviet Math. Dokl. 4 (1963), 1051-1055. MR 27 \#2959.

3. - Some metrization theorems, Uspehi Mat. Nauk 18 (1963), no. 5 (113), 139-145. (Russian) MR 27 \#6242.

4. C. E. Aull, Spaces with a $\sigma$-point-finite base, Proc. Amer. Math. Soc. (to appear).

5. H. R. Bennett, Quasi-developable spaces, Dissertation, Arizona State University, Tempe, Ariz., 1967.

6. C. J. R. Borges, On metrizability of topological spaces, Canad. J. Math. 20 (1968), 795-804. MR 37 \#6910.

7. J. Dugundji, Topology, Allyn and Bacon, Boston, Mass., 1968. MR 33 \#1824.

8. V. V. Fedorčuk, Ordered sets and the product of topological spaces, Vestnik Moskov. Univ. Ser. I Mat. Meh. 21 (1966), 66-71. (Russian) MR 34 \#3515.

9. V. V. Filippov, On feathered paracompacta, Dokl. Akad. Nauk SSSR 178 (1968), 555-558 = Soviet Math. Dokl. 9 (1968), 161-164. MR 37 \#3519.

10. L. Gillman and M. Henricksen, Concerning rings of continuous functions, Trans. Amer. Math. Soc. 77 (1954), 340-362. MR 16, 156.

11. D. W. Hall and G. L. Spencer, Elementary topology, Wiley, New York; Chapman \& Hall, London, 1955. MR 17, 649.

12. S. Hanai, Open mappings and metrization theorems, Proc. Japan Acad. 39 (1963), 450-454. MR 29 \#2772. 
13. R. W. Heath, Screenability, pointwise paracompactness and metrization of Moore spaces, Canad. J. Math. 16 (1964), 763-770.

14. - - On spaces with point-countable bases, Bull. Acad. Polon. Sci. Sér. Sci. Math. Astronom. Phys. 13 (1965), 393-395. MR 32 \#656.

15. - Semi-metric spaces and related spaces, Arizona State University Topology Conference Journal, 1967, pp. 153-161.

16. J. L. Kelley, General topology, Van Nostrand, Princeton, N. J., 1955. MR 16, 1136.

17. H. J. Kowalsky, Topological spaces, Academic Press, New York, 1965.

18. D. J. Lutzer and H. R. Bennett, Separability, the countable chain condition and the Lindelöf property in linearly orderable spaces, Proc. Amer. Math. Soc. 23 (1969), 664-667. MR 40 \#2013.

19. L. F. McAuley, A relation between separability, completeness and normality in semi-metric spaces, Pacific J. Math. 6 (1956), 315-326. MR 18, 325.

20. A. Mišenko, Spaces with a point-countable base, Dokl. Akad. Nauk SSSR 144 (1962), 985-988 = Soviet Math. Dokl. 3 (1962), 855-858. MR 25 \#1537.

21. R. L. Moore, Foundations of point set theory, rev. ed., Amer. Math. Soc. Colloq. Publ., vol. 13, Amer. Math. Soc., Providence, R. I., 1962. MR 27 \#709.

22. V. I. Ponomarev, Metrizability of a finally compact p-space with a point-countable base, Dokl. Akad. Nauk SSSR 174 (1967), 1274-1277 =Soviet Math. Dokl. 8 (1967), 765-768. MR 35 \#7298.

23. M. E. Rudin, Souslin's conjecture, Amer. Math. Monthly 10 (1969), 11131119.

24. L. A. Steen, $A$ direct proof that a linearly ordered space is hereditarily collectionwise normal, Proc. Amer. Math. Soc. 24 (1970), 727-728.

Texas Tech University, Lubbock, Texas 79409 